\title{
Star-forming Substructure within Molecular Clouds
}

\author{
James Di Francesco \\ National Research Council of Canada, 5071 West Saanich Road, \\ Victoria, BC, Canada V9E 2E7 \\ email: james.difrancesco@nrc-cnrc.gc.ca
}

\begin{abstract}
Wide-field far-infrared/submillimeter continuum maps of molecular clouds by the Herschel Space Observatory GBS and HOBYS surveys are revealing the star-forming substructures that lead to star formation in dense gas. In particular, these maps have revealed the central role in clouds of filaments, likely formed through turbulent motions. These filaments appear to be non-isothermal and fragment into cores only when their column densities exceed a stability threshold. Organizations of filament networks suggest the relative role of turbulence and gravity can be traced in different parts of a cloud, and filament intersections may lead to larger amounts of mass flow that form the precursors of high-mass stars or clusters.
\end{abstract}

Keywords. ISM: dust, extinction - ISM: structure — ISM: evolution — submillimeter

\section{Introduction}

Over the last ten years, it has become apparent that star formation in galaxies proceeds not just in molecular gas, but in dense molecular gas. For instance, Wu et al. (2005) (cf. Gao \& Solomon 2004) noted a power-law relationship between $L_{I R}$, the infrared luminosity of extragalactic and galactic sources, with $L_{H C N}$, the luminosity of the HCN $J=1-0$ line, over $\sim 10$ orders of magnitude. Moreover, Lada et al. (2010) found a power-law relationship between the numbers of YSOs in nearby molecular clouds and the amount of material in those clouds with column density over $120 \mathrm{M}_{\odot} \mathrm{pc}^{-2}$. Similarly, Gutermuth et al. (2011) found a power-law correlation between the local surface densities of YSOs and the column density, there measured from extinction. The focus of how stars form in galaxies now seems less about how molecular gas forms in galaxies and more about how dense gas itself arises within molecular clouds.

Dense gas in molecular clouds can be well traced by the thermal continuum emission of dust mixed with that gas. In years past, ground-based submillimeter telescopes have used bolometer arrays to map the dense substructure that arises in such clouds, i.e., starless and protostellar "cores" (see Di Francesco et al. 2007 for a review). Such investigations, however, have been limited by sensitivity but importantly also by the spatial filtering imposed by having to remove a contribution of emission by atmosphere itself. In effect, the dynamic range of such ground-based observations was limited on angular scales by the magnitude of the "chop throw" of the respective secondary mirror. This limitation biased the maps to reveal the brighter, compact substructures within molecular clouds, but not so much the context within which such substructures arose.

In this contribution, we highlight the revolutionary advances recently made in determining the origins of substructure in molecular clouds, and hence star formation itself, made possible with the Herschel Space Observatory (HSO; Pilbratt et al. 2010). In particular, we describe the early results from two Key Programmes, the Herschel Gould Belt Survey (GBS; PI: Ph. André) and the Herschel OB Young Stellar objects survey 
(HOBYS; PI: F. Motte). These surveys consist of large-scale mapping of 15 molecular clouds each over $\sim 1-10 \mathrm{deg}^{-2}$ each in continuum emission using the PACS and SPIRE instruments (see André et al. 2010 and Motte et al. 2010). The GBS concentrates on clouds at distances of $<0.5 \mathrm{kpc}$ while the HOBYS survey concentrates on clouds at $0.5-3.0 \mathrm{kpc}$.

With both instruments working in parallel, the GBS/HOBYS maps yield continuum data at $70 \mu \mathrm{m}$ and $160 \mu \mathrm{m}$ (PACS; Poglitsch et al. 2010) and at $250 \mu \mathrm{m}$, $350 \mu \mathrm{m}$, and $500 \mu \mathrm{m}$ (SPIRE; Griffin et al. 2010) simultaneously at angular resolutions of $\sim 18^{\prime \prime}(\lambda / 250 \mu \mathrm{m})$ FWHM. Located at the Sun-Earth L2 point, HSO observations proceed without needing to subtract an atmospheric signal. Hence, these continuum maps have extremely high sensitivity and spatial dynamic range. Moreover, the wavelength range of PACS and SPIRE covers the peak of SEDs of colder dust at 10-30 K, allowing line-of-sight dust temperatures to be determined. From these, column density maps can be made using the flux of the optically-thin continuum emission itself (assuming the dust opacity $\kappa_{\nu}$ is well known). The resulting high-resolution column density maps provide key insights into how dense, star-forming substructures arise within molecular clouds.

\section{Filaments}

The most important result stemming from the GBS and HOBYS surveys so far has been the recognition that filaments within molecular clouds are central to the formation of more compact, denser cores. Figure 1 shows the column densities obtained for the Aquila Rift cloud by the GBS (Könyves et al. 2010; André et al. 2010). The left panel shows the total column densities toward the cloud overlaid with symbols denoting the locations of prestellar cores and protostars. The right panel shows column densities of the same cloud on "intermediate" spatial scales, i.e., a curvelet component of a spatial

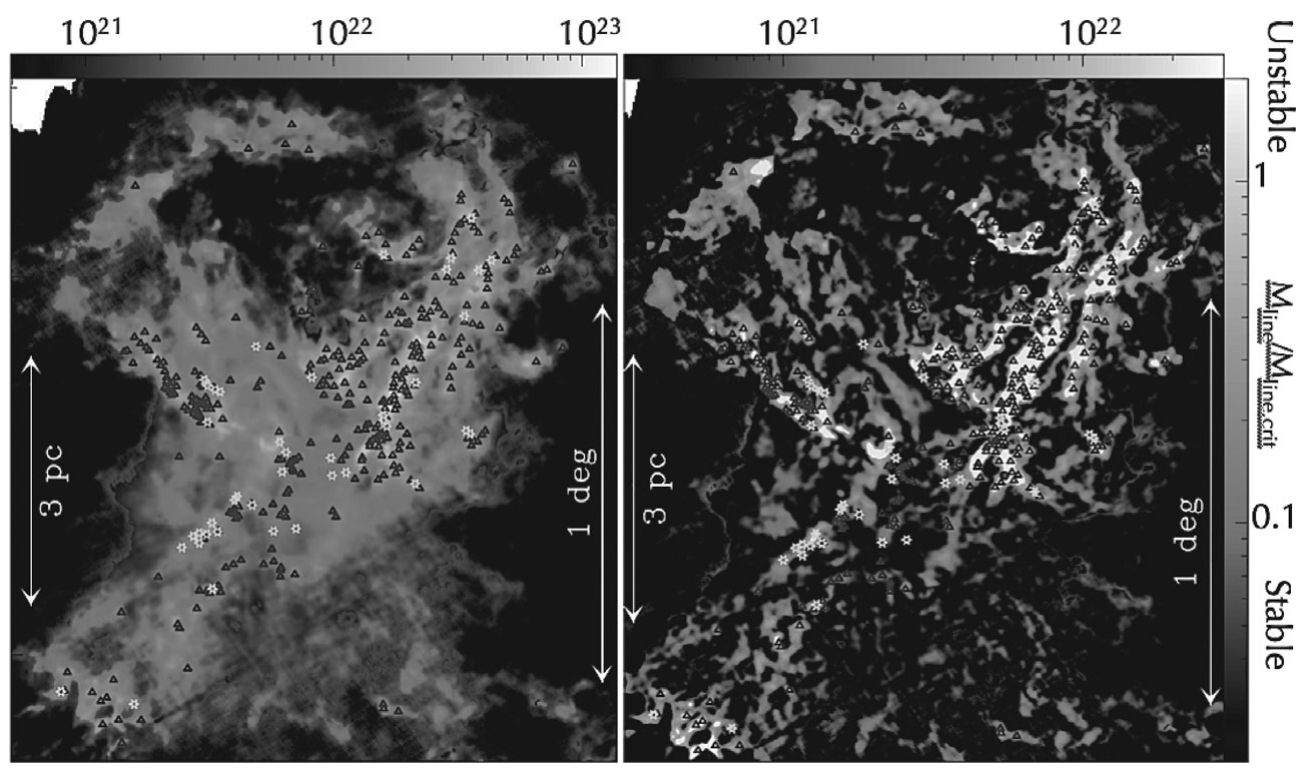

Figure 1. Column density maps toward the Aquila Rift cloud. Triangles and stars denote the locations of prestellar cores and protostars respectively. Left: Total column densities originally from Könyves et al., A\&A, 518, L106, 2010, reproduced with permission @ ESO. Right: Curvelet component of column densities on intermediate scales determined from a spatial decomposition originally from André et al. A\&A, 518, L102, 2010, reproduced with permission (c) ESO. The color scale is chosen so that all regions in white exceed the critical mass per unit length of an isothermal cylinder by Inutsuka \& Miyama (1997). 
decomposition of the column densities seen in the right panel where small- and large-scale structure have been removed. As can be immediately seen, the cloud is threaded everywhere by long, linear structures on these scales, i.e., filaments. Star-forming structures like cores, however, are only located within certain filaments (shaded white in Figure 1) where the mass per unit length exceeds a critical threshold of stability defined for an isothermal cylinder by Inutsuka \& Miyama (1997), i.e., $\mathrm{M}_{\text {line }}=\left(2 c_{s}^{2}\right) / \mathrm{G} \sim 15 \mathrm{M}_{\odot} \mathrm{pc}^{-1}$ at $T=10 \mathrm{~K}$. In other words, filaments are seen to form ubiquitously within molecular clouds, but only those with high enough local column density are susceptible to fragmentation into smaller-scale objects like dense cores. This threshold, equivalent to $A_{V}=6$, neatly explains the extinction or column density thresholds for star formation noted by Lada et al. and others. Indeed, as noted by André et al., the mass distribution of the prestellar cores in the Aquila Rift is similar in morphology to the stellar Initial Mass Function, suggesting that the process of filament formation and fragmentation into cores is related substantially to that of star formation itself.

How do these filaments form? Their ubiquity in the Aquila Rift, and in fact all other clouds analyzed so far by the GBS and HOBYS surveys, is suggestive a process acting globally within molecular clouds. Indeed, a surprising quality of the filaments themselves found by HSO data, namely that they have nearly constant widths, may provide an important clue about their origins. In more recent papers, the GBS and HOBYS surveys have been using an automated algorithm called DisPerSE developed by Sousbie (2011) to trace the cosmic web on cosmological scales to identify filaments within total column density maps. (Filaments identified with DisPerSE have a high degree of correspondence with those seen in spatial component maps like Figure 1, right). Figure 2 shows a

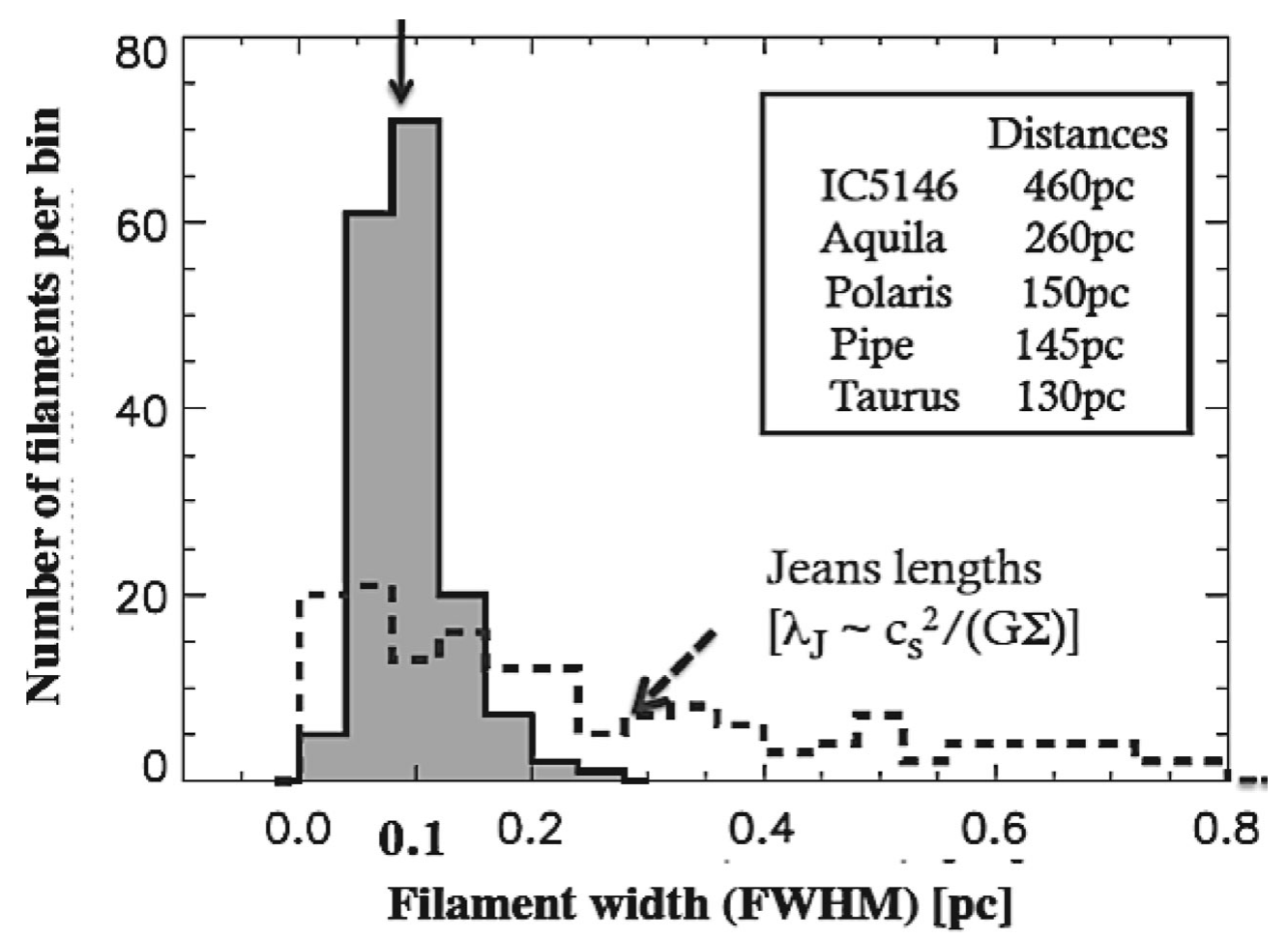

Figure 2. Histogram of the widths of filaments in five nearby molecular clouds determined from Gaussian fitting their central shapes (solid line) and histogram of the thermal Jeans lengths from the same clouds (dashed line) from Arzoumanian et al. 2012, in prep. 
histogram of the Gaussian widths of filaments identified by DisPerSE in the total column density maps of five molecular clouds studied by the GBS, determined by Arzoumanian et al. (2011, 2012, in prep.). Despite the wide range of distances to these clouds and nearly two orders of magnitude in central column density (note the distribution of Jeans lengths denoted by the dashed histogram), the filaments within these clouds have a distribution of width sharply peaked at $0.1 \mathrm{pc}$.

Such common widths may be understood if we regard filaments as shocks between turbulent cells within molecular clouds. As Arzoumanian et al. (2011) note, the thickness of a post-shock region, $\lambda$, can be regarded as the ratio of the thickness of the pre-shock gas, $L$, to the compression ratio of the shock, i.e., the square of the Mach number, $M(L)^{2}$. Since $M(L) \propto L^{0.5}$ from Larson (1981), $\lambda$ is constant. Indeed, $\lambda \approx 0.1 \mathrm{pc}$, can be identified as the sonic scale, i.e, where the magnitude of the turbulent $3 \mathrm{D}$ velocity dispersion equals the sound speed. Furthermore, filaments may keep their widths constant after formation if they evolve in quasi-virial balance. Namely, gas within a filament contracting from its own gravity is replaced by gas accreted from the filament surroundings. The contracting gas releases potential energy, allowing the internal velocities, $\sigma_{v}$, the central column density, $\Sigma_{o}$, and the virial mass per unit length, $\mathrm{M}_{\text {line,vir }}$, in the filament to increase. If $\mathrm{M}_{\text {line,vir }}=\left(2 \sigma_{v}^{2}\right) / \mathrm{G}$, then $\lambda=\mathrm{M}_{\text {line,vir }} /\left(2 \Sigma_{o}\right)$ is approximately constant if $\Sigma_{o} \propto \sigma_{v}^{2}$, as was observed by Heyer et al. (2009) in several Galactic clouds.

Arzoumanian et al. (2011) and more recently Palmerim et al. (2012) also examined the radial structure of filaments in IC 5146 and Taurus B211, respectively. In both cases, they determined a mean profile by averaging all profiles along the filaments and fit the mean profiles assuming an idealized model of a cylindrical filament with density as a function of radius of the form:

$$
\rho_{p}(r)=\frac{\rho_{c}}{\left[1+\left(r / R_{\text {flat }}\right)^{2}\right]^{p / 2}} .
$$

where $\rho_{c}$ is the central density of the filament and $R_{\text {flat }}$ is the characteristic radius of the flat inner portion of the density profile. With this model, the case of an isothermal filament in hydrostatic equilibrium corresponds to $p=4$ and $R_{\text {flat }}$ is equal to the thermal Jeans length at the filament center (Ostriker 1964). Fitting the mean filament profiles, however, these authors find $1.5<p<3$, with none having the steep $p=4$ profile of an isothermal cylinder. The shallow profiles of the actual filaments highlight the fact that they are actually non-isothermal bodies, with temperatures decreasing toward the filament centers. Indeed, Palmerim et al. (2012) find that the temperature drop in B211 can be fit well assuming the filament has an equation of state $P \propto \rho_{P}^{\gamma}$ with $\gamma<1$ (i.e., $0.97 \pm 0.01)$ and $T(r) \propto \rho_{P}(r)^{(\gamma-1)}$. The contribution of magnetic fields to filament structure (e.g., Fiege \& Pudritz 2000) remains to be clarified.

\section{Networks of Filaments}

Though filaments appear directly related to the formation of smaller-scale substructure in clouds like cores, the spatial organization of filaments within a given cloud also appears to influence the masses of the cores formed and perhaps those of the stars formed as well. These insights come from studies of filament networks within various clouds, also made possible by HSO observations for the first time.

In some cases, the degree of uniformity in filament direction may indicate the relative importance of turbulence and gravity within various regions of a cloud. For example, Hill et al. (2011) noted in the HOBYS maps of the Vela C cloud that filament networks were either relatively disorganized into "nests" or highly organized into linear "ridges." 
The nests were noted for their sparse, low-mass star formation but the ridges (e.g., the one containing RCW36) were noted for their massive dense cores and their clustered, high-mass star formation. Indeed, nests may be regions of a cloud where turbulence still has significant influence, and has offset the formation of highly dense structures. Ridges, however, may be locations of high column density $\left(A_{V}>100\right)$, where self-gravity has overwhelmed turbulence and formed a compressed linear structure of relatively high density from which massive stars may form.

In other cases, the degree to which filaments intersect may influence how star formation may be enhanced and how massive stars form in a cloud. For example, Hennemann et al. (2012) noted that the HOBYS maps of the high-mass DR21 ridge of the Cygnus X cloud reveal it to be intersected by several smaller "sub-filaments" that may be in the process of merging with the dominant ridge. Furthermore, Peretto et al. (2012) saw in the GBS maps of the Pipe Nebula that the low-mass B59 region, indeed the only location of star

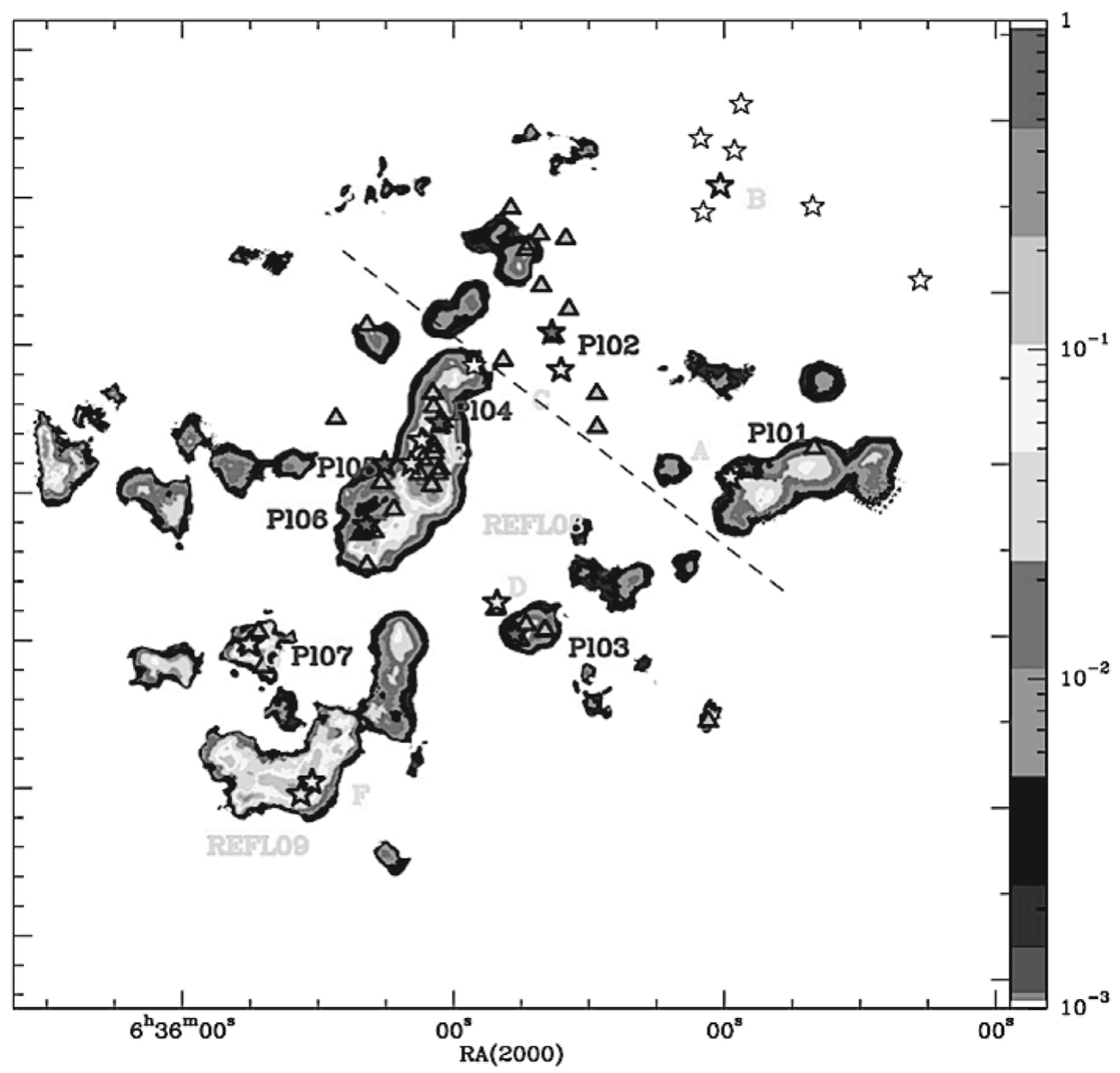

Figure 3. Map of the "confidence" (coded from 0 to 1) of finding cluster formation sites in the Rosette Molecular Cloud made by multiplying the column density map with a mask produced by placing $3^{\prime}$ FWHM Gaussians at the locations of each junction point, and then normalizing from the resultant maximum (originally from Schneider et al., A\&A, 540, L11, 2012, reproduced with permission (C) ESO.). Stars denote the locations of massive stars or dense clumps and triangles denote the locations of infrared clusters (also labeled). 
formation in the cloud, itself has a hub-filament structure. In both cases, the adjoining filaments may be feeding the dominant clump with more mass than would be available to it from a single parent filament, increasing the local density and promoting more star formation.

The picture of enhanced star formation at filament intersections was further quantified recently by a study of HOBYS observations of the Rosette Molecular Cloud by Schneider et al. (2012). There, filaments were identified in the total column density map using DisPerSE and the locations where filaments intersected were found. Schneider et al. then determined the "confidence" of finding infrared clusters and massive dense cores at the locations of filament junctions. This metric was obtained by multiplying the column density map of the RMC with a mask made by placing $3^{\prime}$ FWHM Gaussians at the locations of all filament junctions. Figure 3 shows the results of this test, where a high degree of correspondence is seen between the locations of infrared clusters and massive dense cores (noted as stars or triangles) with filament intersections. In total, this result supports the view that mass flows along filaments and infrared clusters and massive dense cores form at filament intersections where more avenues of mass flow are available. Studies of the kinematics of the gas within these filaments are needed to substantiate this idea further.

\section{Further Work}

The aforementioned studies are only the first results from the rich GBS and HOBYS datasets. Further work, in particular comparing the populations of several clouds, should provide even deeper insights on the origins of substructure within molecular clouds.

\section{References}

Arzoumanian, D., et al. 2011, A\&A, 529, L6

André, Ph., et al. 2010, A\&A, 518, L102

Di Francesco, J., Evans, N. J., II, Caselli, P., Myers, P. C., Shirley, Y., Aikawa, Y., \& Tafalla, M. 2007, in: B. Reipurth, D. Jewitt, \& K. Keil (eds.) Protostars and Planets V (Tucson: University of Arizona Press), p. 17

Fiege, J. D. \& Pudritz, R. E. 2000, MNRAS, 311, 85

Gao, Y. \& Solomon, P. M. 2004, ApJ, 606, 271

Griffin, M., et al. 2010 A\& A, 518, L3+

Gutermuth, R. A., et al., 2011 ApJ, 739, 84

Hennemann, M., et al. 2012, A\&A, 543, 3

Heyer, M., Krawczyk, C., Duval, J., \& Jackson, J. M., 2009, ApJ, 699, 1092

Hill, T., et al. 2011, A\& A, 533, 94

Inutska, S.-I., \& Miyama, S. M. 1997 ApJ, 480, 681

Könyves, V., et al., 2010, A\&SA, 518, L106

Lada, C. J., Lombardi, M., \& Alves, J. F. 2010, ApJ, 724, 687

Larson, R. B. 1981, MNRAS, 194, 809

Motte, F., et al. 2010, A\&A A, 518, L77

Ostriker, J. 1964, ApJ, 140, 1056

Palmerim, P., et al. $2012 A \& A$, submitted

Peretto, N., et al. 2012, A\&A, 541, 63

Pilbratt, G., et al. 2010, A\&SA, 518, L1+

Poglitsch, A., et al. 2010, A\&SA, 518, L2+

Schneider, N., et al. 2012, A\&SA, 540, L11

Sousbie, T. 2011, MNRAS, 414, 350

$\mathrm{Wu}$, J., et al. 2005, 635, L173 